\title{
ESTUDO DE VIABILIDADE DE INVESTIMENTO ATRAVÉS DO PAYBACK, VPL E TIR
}

\author{
Alexandre Pioner de Lima ${ }^{1}$ \\ Miguelina Troisi Bonfrisco ${ }^{2}$
}

\section{Resumo}

Existem várias ferramentas para verificar o retorno de um investimento. Este artigo irá discorrer sobre três indicadores, que associados, irão auxiliar nesta tomada de decisão. A avaliação do investimento será feita através do VPL (Valor Presente Líquido), TIR (Taxa Interna de Retorno) e Payback (Tempo de Retorno). O VPL é um indicador que analisa todos os fluxos de caixa esperados pelo investimento em uma data e, de seu total, subtrai-se o valor inicial de investimento. A TIR representa a rentabilidade de um projeto de investimento, comparada a um investimento no mercado financeiro. $O$ Payback mede quanto tempo um projeto levará para gerar os retornos ou lucros que paguem o investimento inicial. Quanto a sua natureza, a pesquisa será aplicada, objetivando produzir conhecimentos para aplicação prática, dirigidos à solução de problemas específicos. Quanto à abordagem do problema, a pesquisa será quantitativa, traduzindo-se em números as informações para classificá-las e analisá-las. Quanto ao ponto de vista de seus objetivos, a pesquisa será exploratória, com a utilização de pesquisas bibliográficas. Quanto ao ponto de vista dos procedimentos técnicos, a pesquisa será bibliográfica, utilizando material já publicado, constituindo-se basicamente de livros e artigos periódicos, com informações disponibilizadas na internet.

Palavras-chave: Investimento, VPL, TIR, Payback.

\section{INVESTMENT FEASIBILITY STUDY THROUGH PAYBACK, NPV AND IRR}

\begin{abstract}
There are several tools for verifying the return on an investment. This article will discuss three indicators, which associated, will assist in this decision making. The valuation of the investment will be made through NPV (Net Present Value), IRR (Internal Rate of Return) and Payback (Return Time). NPV is an indicator that analyzes all expected cash flows from the

\footnotetext{
${ }^{1}$ Mestre em Administração. PUC-Rio - Administrador do Serviço de Orçamento do Hospital de Clínicas de Porto Alegre. aplima@ hcpa.edu.br

${ }^{2}$ Pós-Graduação em Administração Financeira. UNIASSELVI - Chefe do Serviço de Finanças do Hospital de Clínicas de Porto Alegre. mbonfrisco@hcpa.edu.br
} 
investment on a date and, accordingly, total is subtracted from initial investment value. IRR represents the profitability of an investment project compared to an investment in the financial market. Payback measures how long it will take a project to generate the returns or profits that pay off the initial investment. The research will be applied, aiming to produce knowledge for practical application, directed to the solution of specific problems. As for the approach of the problem, the research will be quantitative, translating information is given in numbers to classify and analyze them. Regarding the point of view of its objectives, the research will be exploratory, using bibliographic research. Regarding the point of view of technical procedures, the research will be bibliographic, using material already published, consisting basically of books and periodical articles, with information available on the Internet.

Keywords: Investment, NPV, IRR, Payback.

\section{INTRODUÇÃO}

Uma atividade importante para o funcionamento de uma empresa é o processo de investimento em ativos. Para tanto, a empresa deve avaliar se este investimento terá ou não o retorno esperado, ou decidir entre vários projetos. Isso é fundamental porque o dinheiro é caro e não se pode desperdiçá-lo.

Este artigo foi elaborado para auxiliar e orientar os investidores de como realizar um estudo de viabilidade de investimento, a fim de reduzir os riscos. Para tanto, iremos fazer um estudo de caso, através do qual iremos aplicar três técnicas de análise de investimento: o VPL, a TIR e o Payback.

A primeira parte do estudo é determinar o que se vai comprar e apurar o custo total desta aquisição. O segundo passo é fazer um fluxo de caixa do projeto. Determinar todos os dispêndios financeiros e todas as receitas oriundas deste investimento. Após isso, apurar o lucro (ou prejuízo).

Com esses dados, começa-se o estudo de viabilidade de investimento. 


\section{OBJETO DO INVESTIMENTO}

Para o estudo de viabilidade de investimento, vamos eleger a aquisição de um aparelho de ecografia para uma clínica médica ou para um hospital. A forma de compra será uma importação. Ao valor do equipamento deverá ser acrescido todos os custos relacionados com esta compra, seja despesas aduaneiras, tributos federais, armazenagem, transporte aéreo, marítimo e/ou rodoviário, ISSQN, ICMS, remessas de DOC, capatazia, taxa SISCOMEX, débito de LI ANVISA, taxa de desconsolidação, delivery, collect, seguro e demais despesas.

Em outubro de 2019, o valor médio de um ecógrafo, acrescido dos custos, é em torno de US\$ 152.916,41. O valor médio do dólar nesta data é de R \$ 4,21 (Fonte: Banco Central do Brasil - Indicadores). Isso resulta em um custo de aquisição de $\mathrm{R} \$ 643.778,08$.

Estima-se que este equipamento tenha um retorno do investimento em 24 meses, ou 02 anos. Ao mesmo tempo, espera-se um resultado superior à taxa de 0,5492 ao mês, valor médio do CDB (Fonte: Zero Hora - Indicadores).

\section{APURAÇÃO DAS DESPESAS}

Para a elaboração do Fluxo de Caixa faz-se necessário apurar todas as despesas relacionadas com a clínica médica ou hospital. Começamos com o item que representa o maior gasto mensal: despesas de pessoal. Devemos apurar todos os dispêndios com salários, $13^{\circ}$ salário, férias, adicional noturno, horas extras, insalubridade, função gratificada, encargos de FGTS, encargos de INSS e demais despesas.

No Fluxo de Caixa, apurar todas as despesas com materiais de consumo e medicamentos, como também depreciação de bens móveis e imóveis. No item de despesas gerais, apurar gastos com locomoção urbana, locação de bens móveis, manutenção de máquinas e equipamentos, seguros em geral, limpeza e conservação, contribuição sindical, contribuição patronal de previdência social, auxílio creche, vale transporte, vale refeição, assistência médica e odontológica, e demais despesas.

Para o exemplo, apuramos a seguinte tabela:

\begin{tabular}{l|l|r}
\hline & Composição Custo Direto & Valor \\
\hline Pessoal Próprio & Salários & $191.001,24$ \\
& Descontos de Salários & $(23.470,50)$ \\
& $13^{\circ}$ Salário & $6.126,73$
\end{tabular}




\begin{tabular}{l|l|r} 
& Adicional Noturno & $25.295,91$ \\
& Função Gratificada & $15.615,55$ \\
& Vale Transporte & $18.333,85$ \\
& Insalubridade & $28.168,42$ \\
& Horas Extras & $1.191,05$ \\
& Encargos FGTS & $21.742,92$ \\
& Encargos INSS & $78.304,17$ \\
\hline & Total & $\mathbf{5 1 . 7 5 8 , 4 8}$ \\
\hline Material Consumo & Medicamentos & 161,80 \\
& Materiais Consumo & $72.827,41$ \\
\hline & Total & $\mathbf{1 0 . 4 2 7 , 0 3}$ \\
\hline Depreciação & Bens Móveis & $6.068,79$ \\
& Bens Imóveis & $3.757,03$ \\
\hline Despesas Gerais & Total & $\mathbf{1 . 4 0 3 , 6 9}$ \\
& Locomoção Urbana & 13,00 \\
& Locação de Bens Móveis & $16.000,00$ \\
& Manutenção e Conservação de Máquinas e Equipamentos & $2.671,00$ \\
& Seguros em Geral & 386,47 \\
& Limpeza e Conservação & 145,48 \\
& Assistência Médica & $19.862,30$ \\
& Contribuição Sindical & 282,43 \\
& Auxílio Alimentação & $42.061,13$ \\
& Vale Transporte & $7.775,97$ \\
& Contribuição Patronal da Previdência Social & $9.166,76$ \\
& Auxílio Creche & $1.067,38$ \\
\hline Total & Total & $\mathbf{1 4 . 2 0 4 , 5 6}$ \\
\hline & & $\mathbf{7 7 . 7 9 3 , 7 6}$ \\
\hline
\end{tabular}

Tabela 1 - Despesas

\section{APURAÇÃO DAS RECEITAS}

Para a apuração das receitas deve-se verificar todas as origens de recursos oriundas da utilização do equipamento. Utiliza-se uma média de exames para a apuração da receita.

\begin{tabular}{l|r|c|c}
\hline & Número de Exames Mensais & Valor Unitário & Valor \\
\hline Convênios & 400 & 200,00 & $80.000,00$ \\
Particular & 80 & 250,00 & $20.000,00$ \\
\hline Total & $\mathbf{4 8 0}$ & & $\mathbf{1 0 0 . 0 0 0 , 0 0}$ \\
\hline
\end{tabular}

Tabela 2 - Receitas

\section{RESULTADO}

O resultado é um cálculo simples da diferença entre as receitas e as despesas: 


\begin{tabular}{l|r}
\hline & \\
\hline Receitas & $100.000,00$ \\
Despesas & $77.793,76$ \\
\hline Resultado & $\mathbf{2 2 . 2 0 6 , 2 4}$ \\
\hline
\end{tabular}

Tabela 3 - Resultado

Com essas informações, iniciamos o estudo de viabilidade de investimento através dos três critérios.

\title{
6 PAYBACK
}

O Payback é o mais simples dos critérios para avaliar o investimento. Conforme GITMAN (2010), o período de payback é o tempo necessário para que a empresa recupere o investimento inicial em um projeto, calculado a partir das entradas de caixa.

O cálculo é feito com a seguinte equação:

$$
n_{p}=\frac{I_{0}}{S}
$$

Onde, np é o número de períodos, Io é o investimento inicial e $\mathrm{S}$ é o resultado do fluxo de caixa mensal.

Utilizando a equação, obtemos o resultado de 28,99 meses. Este é o tempo de retorno do investimento. A diretoria da clínica médica ou hospital esperava um retorno de 24 meses.

\begin{abstract}
A principal fragilidade do período de payback está no fato de que o período adequado de payback nada mais é que um número determinado de forma subjetiva. Não pode ser especificado à luz do objetivo de maximização da riqueza, pois não se baseia no desconto de fluxos de caixa para determinar se agregam ao valor da empresa. Em vez disso, o período adequado de payback é apenas o prazo máximo aceitável fixado pela administração e no qual os fluxos de caixa do projeto atingem o break even (isto é, o ponto de equilíbrio com o investimento inicial. (GITMAN, 2010, p.366)
\end{abstract}

\section{VALOR PRESENTE LÍQUIDO - VPL}

O Valor Presente Líquido - VPL é uma técnica de avaliação da viabilidade de um investimento que desconta os fluxos de caixa de um projeto a uma taxa específica. Conforme GITMAN (2010), o valor presente líquido (VPL) é encontrado subtraindo-se o investimento 
inicial de um projeto $(\mathrm{FCo})$ do valor presente de suas entradas de caixa $(\mathrm{FC} 1)$, descontadas à taxa de custo de capital da empresa (r).

O cálculo é feito com a seguinte equação:

$$
N P V=\sum_{t=1}^{n} \frac{S_{t}}{(1+k)^{t}-I_{0}}
$$

Onde, St é o resultado do Fluxo de Caixa Mensal, k é a taxa referencial de aplicação financeira, t é o número de períodos e Io é o investimento inicial.

Utilizando-se no Excel a função VPL, calcula-se a seguinte tabela ao longo do tempo:

\begin{tabular}{c|r|r}
\hline Meses & Fluxo Caixa & VPL \\
\hline 0 & $(\mathrm{R} \$ 643.778,08)$ & \\
1 & $\mathrm{R} \$ 22.206,24$ & $(\mathrm{R} \$ 618.297,64)$ \\
2 & $\mathrm{R} \$ 22.206,24$ & $(\mathrm{R} \$ 596.453,26)$ \\
3 & $\mathrm{R} \$ 22.206,24$ & $(\mathrm{R} \$ 574.728,19)$ \\
4 & $\mathrm{R} \$ 22.206,24$ & $(\mathrm{R} \$ 553.121,77)$ \\
5 & $\mathrm{R} \$ 22.206,24$ & $(\mathrm{R} \$ 531.633,36)$ \\
6 & $\mathrm{R} \$ 22.206,24$ & $(\mathrm{R} \$ 510.262,32)$ \\
7 & $\mathrm{R} \$ 22.206,24$ & $(\mathrm{R} \$ 489.007,99)$ \\
8 & $\mathrm{R} \$ 22.206,24$ & $(\mathrm{R} \$ 467.869,75)$ \\
9 & $\mathrm{R} \$ 22.206,24$ & $(\mathrm{R} \$ 446.846,96)$ \\
10 & $\mathrm{R} \$ 22.206,24$ & $(\mathrm{R} \$ 425.938,99)$ \\
11 & $\mathrm{R} \$ 22.206,24$ & $(\mathrm{R} \$ 405.145,21)$ \\
12 & $\mathrm{R} \$ 22.206,24$ & $(\mathrm{R} \$ 384.465,00)$ \\
13 & $\mathrm{R} \$ 22.206,24$ & $(\mathrm{R} \$ 363.897,74)$ \\
14 & $\mathrm{R} \$ 22.206,24$ & $(\mathrm{R} \$ 343.442,81)$ \\
15 & $\mathrm{R} \$ 22.206,24$ & $(\mathrm{R} \$ 323.099,60)$ \\
16 & $\mathrm{R} \$ 22.206,24$ & $(\mathrm{R} \$ 302.867,50)$ \\
17 & $\mathrm{R} \$ 22.206,24$ & $(\mathrm{R} \$ 282.745,90)$ \\
18 & $\mathrm{R} \$ 22.206,24$ & $(\mathrm{R} \$ 262.734,19)$ \\
19 & $\mathrm{R} \$ 22.206,24$ & $(\mathrm{R} \$ 242.831,79)$ \\
20 & $\mathrm{R} \$ 22.206,24$ & $(\mathrm{R} \$ 223.038,08)$ \\
21 & $\mathrm{R} \$ 22.206,24$ & $(\mathrm{R} \$ 203.352,48)$ \\
22 & $\mathrm{R} \$ 22.206,24$ & $(\mathrm{R} \$ 183.774,40)$ \\
23 & $\mathrm{R} \$ 22.206,24$ & $(\mathrm{R} \$ 164.303,24)$ \\
24 & $\mathrm{R} \$ 22.206,24$ & $(\mathrm{R} \$ 144.938,43)$ \\
25 & $\mathrm{R} \$ 22.206,24$ & $(\mathrm{R} \$ 125.679,39)$ \\
26 & $\mathrm{R} \$ 22.206,24$ & $(\mathrm{R} \$ 106.525,53)$ \\
27 & $\mathrm{R} \$ 22.206,24$ & $(\mathrm{R} \$ 87.476,29)$ \\
28 & $\mathrm{R} \$ 22.206,24$ & $(\mathrm{R} \$ 68.531,08)$ \\
29 & $\mathrm{R} \$ 22.206,24$ & $(\mathrm{R} \$ 49.689,35)$ \\
30 & $\mathrm{R} \$ 22.206,24$ & $(\mathrm{R} \$ 30.950,52)$ \\
31 & $\mathrm{R} \$ 22.206,24$ & $(\mathrm{R} \$ 12.314,04)$ \\
32 & $\mathrm{R} \$ 22.206,24$ & $\mathrm{R} \$ 6.220,65$ \\
33 & $\mathrm{R} \$ 22.206,24$ & $\mathrm{R} \$ 24.654,11$ \\
34 & $\mathrm{R} \$ 22.206,24$ & $\mathrm{R} \$ 42.986,90$ \\
35 & $\mathrm{R} \$ 22.206,24$ & $\mathrm{R} \$ 61.219,56$ \\
36 & $\mathrm{R} \$ 22.206,24$ & $\mathrm{R} \$ 79.352,64$ \\
\hline & & \\
\hline
\end{tabular}


Tabela 4 - VPL

Observa-se que a partir do mês 32, cerca de 02 anos e 08 meses, o projeto de investimento começa a apresentar um retorno financeiro, dado que o resultado do cálculo do VPL é positivo. A diretoria da empresa esperava um retorno de 24 meses.

\section{TAXA INTERNA DE RETORNO - TIR}

Conforme BRAGA (2011), a TIR corresponde a uma taxa de desconto que iguala o valor atual das entradas líquidas de caixa ao valor atual dos desembolsos relativos ao investimento líquido.

O cálculo é feito com a seguinte equação:

$$
\sum_{t=1}^{n} \frac{S_{t}}{(1+R)^{t}}=I_{0}
$$

Onde, St é o resultado do Fluxo de Caixa Mensal, R é a taxa referencial de aplicação financeira, t é o número de períodos e Io é o investimento inicial.

Utilizando-se no Excel a função TIR, calcula-se a seguinte tabela ao longo do tempo:

\begin{tabular}{c|r|c}
\hline Meses & \multicolumn{1}{|c|}{ Fluxo Caixa } & \multicolumn{1}{c}{ TIR } \\
\hline 0 & $(\mathrm{R} \$ 643.778,08)$ & \\
1 & $\mathrm{R} \$ 22.206,24$ & $-96,55 \%$ \\
2 & $\mathrm{R} \$ 22.206,24$ & $-79,62 \%$ \\
3 & $\mathrm{R} \$ 22.206,24$ & $-62,63 \%$ \\
4 & $\mathrm{R} \$ 22.206,24$ & $-49,47 \%$ \\
5 & $\mathrm{R} \$ 22.206,24$ & $-39,65 \%$ \\
6 & $\mathrm{R} \$ 22.206,24$ & $-32,26 \%$ \\
7 & $\mathrm{R} \$ 22.206,24$ & $-26,60 \%$ \\
8 & $\mathrm{R} \$ 22.206,24$ & $-22,17 \%$ \\
9 & $\mathrm{R} \$ 22.206,24$ & $-18,65 \%$ \\
10 & $\mathrm{R} \$ 22.206,24$ & $-15,79 \%$ \\
11 & $\mathrm{R} \$ 22.206,24$ & $-13,45 \%$ \\
12 & $\mathrm{R} \$ 22.206,24$ & $-11,51 \%$ \\
13 & $\mathrm{R} \$ 22.206,24$ & $-9,87 \%$ \\
14 & $\mathrm{R} \$ 22.206,24$ & $-8,48 \%$ \\
15 & $\mathrm{R} \$ 22.206,24$ & $-7,29 \%$ \\
16 & $\mathrm{R} \$ 22.206,24$ & $-6,27 \%$ \\
17 & $\mathrm{R} \$ 22.206,24$ & $-5,38 \%$ \\
18 & $\mathrm{R} \$ 22.206,24$ & $-4,60 \%$ \\
19 & $\mathrm{R} \$ 22.206,24$ & $-3,91 \%$ \\
20 & $\mathrm{R} \$ 22.206,24$ & $-3,30 \%$ \\
21 & $\mathrm{R} \$ 22.206,24$ & $-2,76 \%$
\end{tabular}




\begin{tabular}{l|l|l}
22 & $\mathrm{R} \$ 22.206,24$ & $-2,28 \%$ \\
23 & $\mathrm{R} \$ 22.206,24$ & $-1,85 \%$ \\
24 & $\mathrm{R} \$ 22.206,24$ & $-1,46 \%$ \\
25 & $\mathrm{R} \$ 22.206,24$ & $-1,11 \%$ \\
26 & $\mathrm{R} \$ 22.206,24$ & $-0,79 \%$ \\
27 & $\mathrm{R} \$ 22.206,24$ & $-0,50 \%$ \\
28 & $\mathrm{R} \$ 22.206,24$ & $-0,24 \%$ \\
29 & $\mathrm{R} \$ 22.206,24$ & $0,00 \%$ \\
30 & $\mathrm{R} \$ 22.206,24$ & $0,22 \%$ \\
31 & $\mathrm{R} \$ 22.206,24$ & $0,42 \%$ \\
32 & $\mathrm{R} \$ 22.206,24$ & $0,61 \%$ \\
33 & $\mathrm{R} \$ 22.206,24$ & $0,78 \%$ \\
34 & $\mathrm{R} \$ 22.206,24$ & $0,94 \%$ \\
35 & $\mathrm{R} \$ 22.206,24$ & $1,09 \%$ \\
36 & $\mathrm{R} \$ 22.206,24$ & $1,22 \%$ \\
\hline
\end{tabular}

Tabela 5 - TIR

Observa-se que a partir do mês 32, cerca de 02 anos e 08 meses, o projeto de investimento começa a apresentar um retorno financeiro superior à taxa de aplicação financeira utilizada como referencial, dado que o resultado do cálculo da TIR é positivo. A diretoria da empresa esperava um retorno de 24 meses.

\section{CONSIDERAÇÕES FINAIS}

O primeiro passo para o estudo de viabilidade de investimento é saber exatamente o que se pretende comprar, como também o custo total desta operação. A partir daí, elabora-se um fluxo de caixa, apurando-se todos os dispêndios financeiros relacionados com o investimento, como também a projeção das receitas oriundas deste investimento.

Faz-se necessário, também, estipular um prazo esperado de retorno do investimento, como também comparar a uma taxa de aplicação financeira, caso optasse investir o dinheiro do mercado, ao invés de comprar.

De posse de todos os dados, passe-se à fase do cálculo do retorno. Recomenda-se utilizar uma planilha de cálculo Excel, a qual servirá de base para o estudo de outros investimentos futuros.

Após os cálculos do Payback, do Valor Presente Líquido - VPL e da Taxa Interna de Retorno - TIR, obtivemos os seguintes resultados: 


\begin{tabular}{l|c}
\hline & Retorno em Meses \\
\hline Payback & 28,99 \\
VPL & 32,00 \\
TIR & 32,00 \\
\hline & \\
\hline
\end{tabular}

Tabela 6 - Retorno do Investimento

Observa-se que, salvo uma diferença de 03 meses do Payback, os resultados são semelhantes. Isso vem reforçar a tomada de decisão. Os critérios não são excludentes, mas complementares.

Os três critérios apresentam um mesmo ponto de equilíbrio entre o resultado do fluxo de caixa e as despesas correntes do mês. Demonstra quanto tempo levará para recuperar o investimento inicial, conforme a capacidade de produção e os dispêndios mensais.

No exemplo, como trata-se de um equipamento fundamental para a operação da empresa, outros critérios subjetivos estão relacionados com a tomada de decisão, pois o atendimento ao paciente é o fator mais importante. Os critérios servem para a empresa ter uma visão do custo do dinheiro investido e o tempo do retorno.

Conclui-se que os três critérios nos dão uma visão realista sobre o custo do dinheiro investido e o seu retorno. 


\section{REFERÊNCIAS}

BRAGA, Roberto. Fundamentos e técnicas de administração financeira. São Paulo : Atlas, 2011.

CARVALHO, Juracy Vieira de. Análise econômica de investimentos. Rio de Janeiro : Qualitymark, 2002.

ECO, Umberto. Como se faz uma tese. São Paulo : Perspectiva, 1996.

GALESNE, Alain; FENSTERSEIFER, Jaine E.; LAMB, Roberto. Decisões de investimentos da empresa. São Paulo : Atlas, 1999.

GITMAN, Lawrence J. Princípios de Administração Financeira. 12a Ed. Pearson : São Paulo, 2010.

HÜHNE, Leda Miranda. Metodologia científica. Rio de Janeiro : Agir, 1995.

LEVY, Haim; SARNAT, Marshall. Capital investment \& financial decisions. Cambridge : Prentice Hall, 1997.

ROSS, S. A.; WESTERFIELD, R. W.; JAFFE, J. F. Administração financeira. São Paulo : Atlas, 1995.

SEVERINO, Antonio Joaquim. Metodologia do trabalho científico. São Paulo : Cortez, 1996.

STEVENSON, William J. Estatística aplicada à administração. São Paulo : Harper \& Row do Brasil, 1981.

TOSI, Armando José. Matemática financeira com utilização do Excel® 2010. São Paulo : Atlas, 2012. 